\title{
钙铁矿单晶薄膜生长-转移新策略
}

\author{
邹贵付
}

苏州大学能源学院, 能源与材料创新研究院, 江苏省先进碳材料与可穿戴能源技术重点实验室, 苏州 215006

E-mail: zouguifu@suda.edu.cn

\section{A growth-transfer strategy for perovskite single-crystal thin films}

\section{Guifu Zou}

Key Laboratory of Advanced Carbon Materials and Wearable Energy Technologies of Jiangsu Province, Soochow Institute for Energy and Materials Innovations, College of Energy, Soochow University, Suzhou 215006, China

E-mail: zouguifu@suda.edu.cn

doi: 10.1360/TB-2020-0990

卤素钙钠矿材料是近些年来被广泛关注的一类材料, 这得益于其优异的光电特性, 例如高光吸收系数、长载流 子扩散长度、带隙可调等, 这些特性使得其在太阳能电池、 高清显示、探测器等领域有着巨大的应用前景 ${ }^{[1 \sim 4]}$. 相比于
钙钛矿多晶薄膜，单晶钙钛矿的定向传输性质和低缺陷浓 度使得其具有更优异的载流子输运性能和稳定性 ${ }^{[59]}$. 目 前所报道的制备单晶钻钠矿材料的方法主要有空间限域 法、气相外延生长法、表面张力辅助生长法、自上而下生 (a)

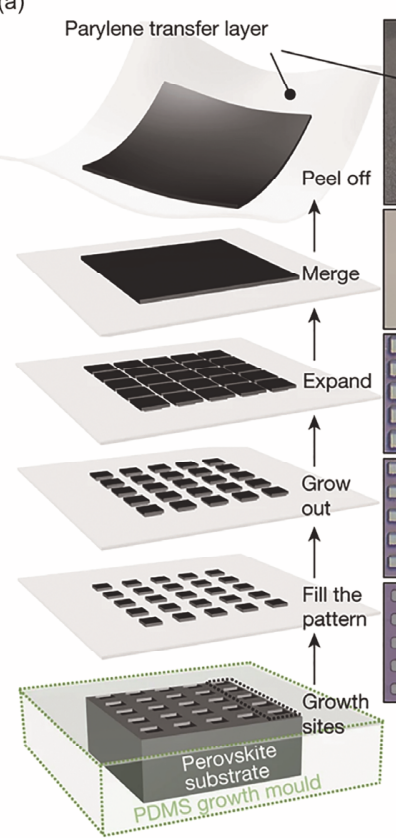

(b)

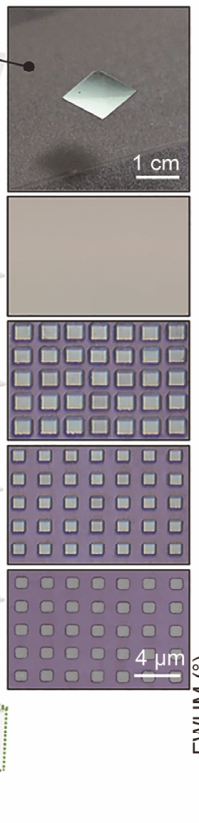

(d)
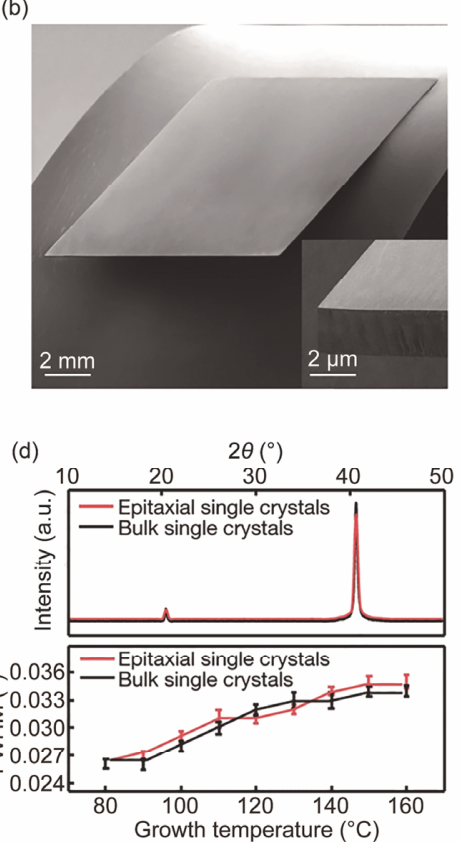

(c)

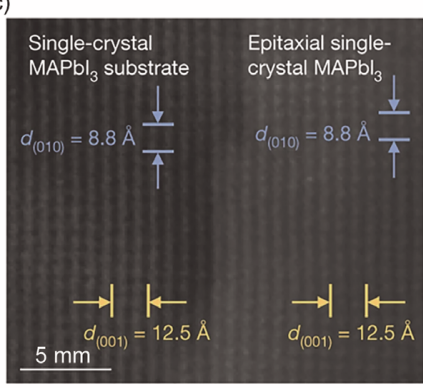

(e)

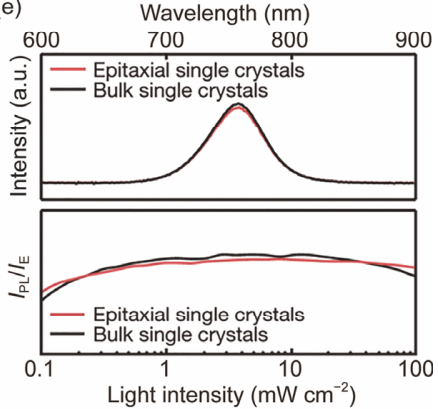

图 1 (网络版彩色)光刻法辅助的基于溶液外延生长和转移方法制备高质量的单晶杂化钙钛矿薄膜. (a) 单晶 $\mathrm{MAPbI}_{3}$ 薄膜的合成过程; (b) 单 晶 $\mathrm{MAPbI}_{3}$ 薄膜的 $\mathrm{SEM}$ 图像; (c) 单晶 $\mathrm{MAPbI}_{3}$ 的 TEM图像; (d) 钻针矿块材单晶及转移的外延单晶薄膜的XRD对照图; (e) 钙钛矿块材单晶及转 移的外延单晶薄膜的PL和PL强度对照图 ${ }^{[15]}$

Figure 1 (Color online) The lithography-assisted epitaxial-growth-and-transfer method for fabricating high-quality, single-crystal hybrid perovskite thin films. (a) Synthetic process of the single-crystal perovskite thin film; (b) SEM images of single-crystal MAPbI ${ }_{3}$ thin films; (c) TEM image of homo-epitaxial single-crystal $\mathrm{MAPbI}_{3}$ thin films; (d) XRD comparison between the bulk single-crystal and the transferred epitaxial single-crystal thin film; (e) PL and PL intensity comparisons between bulk single crystals and transferred epitaxial single-crystal thin films ${ }^{[15]}$ 
长法, 空化触发不对称结晶法等 ${ }^{[10 ~ 14]}$. 然而, 由于晶体的 结晶总是发生在气-液界面处, 这会干扰到后续晶体的长 大. 此外, 钙钛矿层梯度变化的带隙能够实现光的充分吸 收和载流子更有效的捕获, 但是目前还没有相关的单晶钙 钛矿组分梯度生长的报道. 因此, 单晶钙钛矿形貌与组分 的可控合成是具有挑战性的.

近日, 针对这些问题, 美国加州大学圣地亚哥分校徐 升课题组 ${ }^{[15]}$ 在Nature 发表了题为 “A fabrication process for flexible single-crystal perovskite devices”的文章, 他们开发 了一种光刻法辅助的基于溶液外延生长和转移单晶钙钛矿 的策略, 制备流程如图1(a)所示. 通过光刻技术对覆盖在单 晶钙钛矿块材的掩膜上进行掩膜图案的刻蚀, 在溶液法生 长过程中, 最初晶体的成核发生在衬底的暴露面处, 随着 晶体的外延生长溢出掩膜深度, 多个小单晶在横向上合并 成单晶薄膜. 扫面电子显微镜 (SEM) 图像与高分辨透射电 子显微镜(TEM)图像证明了所生长的单晶薄膜不存在晶界 并且存在与衬底的外延关系. 同时, 该单晶薄膜能够被转 移至任意基底上, 并保持高结晶度和与基底良好的附着力.
基于这种方法, 可以通过掩膜图案的设计实现单晶厚 度的精确控制 $(600 \mathrm{~nm} \sim 100 \mu \mathrm{m}$ ) 和大面积的制备(最大约为 $5.5 \mathrm{~cm} \times 5.5 \mathrm{~cm})$. 同时, 通过供给不同组分的前驱体溶液还 可实现单晶厚度方向上的组分调控(例如从 $\mathrm{MAPbI}_{3}$ 过渡到 $\mathrm{MAPb}_{0.5} \mathrm{Sn}_{0.5} \mathrm{I}_{3}$ ), 梯度的铅-锡合金化形成了梯度变化的带 隙, 进一步提升了载流子迁移率和抑制载流子复合. 基于 铅-锡梯度结构的单晶钙钛矿光伏电池获得平均 $18.77 \%$ 的 光电转换效率, 并表现出相比于多晶电池更优异的长期稳 定性和光热稳定性.

该团队实现了钙钛矿单晶薄膜的精确可控生长, 解决 了目前单晶厚度(低于微米级)和大面积不能双赢的难点, 并首次合成了组分梯度变化的单晶, 基于该梯度结构的单 晶太阳能电池表现出高稳定性. 重要的是, 该生长技术是 基于传统半导体制造的光刻技术，能够实现与现有工业流 程很好的兼容性, 这对于单晶钙钛矿材料的商业化应用具 有重要意义. 未来进一步的简化器件制备工艺, 包括避免 材料转移或者实现钲钛矿单晶的原位生长, 则是亟待解决 的关键问题.

\section{参考文献}

1 Yang W S, Park B W, Jung E H, et al. Iodide management in formamidinium-lead-halide-based perovskite layers for efficient solar cells. Science, 2017, 356: 1376-1379

2 Lin K, Xing J, Quan L N, et al. Perovskite light-emitting diodes with external quantum efficiency exceeding 20\%. Nature, 2018, 562: $245-248$

3 Feng J, Gong C, Gao H, et al. Single-crystalline layered metal-halide perovskite nanowires for ultrasensitive photodetectors. Nat Electron, 2018, 1: 404-410

4 US Department of Energy. National Renewable Energy Laboratory. Best Research-Cell Efficiency Chart. 2020. https://www.nrel.gov/pv/ cell-efficiency.html

5 Leblebici S Y, Linn L, Li Y, et al. Facet-dependent photovoltaic efficiency variations in single grains of hybrid halide perovskite. Nat Energy, 2016, 1: 16093

6 Zhang W, Pathak S, Sakai N, et al. Enhanced optoelectronic quality of perovskite thin films with hypophosphorous acid for planar heterojunction solar cells. Nat Commun, 2015, 6: 10030

7 Zheng G, Zhu C, Ma J, et al. Manipulation of facet orientation in hybrid perovskite polycrystalline films by cation cascade. Nat Commun, 2018, 9: 279

8 Chen Z, Dong Q, Liu Y, et al. Thin single-crystal perovskite solar cells to harvest below-bandgap light absorption. Nat Commun, 2017, 8: 1890

9 Lee L, Baek J, Par K S, et al. Wafer-scale single-crystal perovskite patterned thin films based on geometrically-confined lateral crystal growth. Nat Commun, 2017, 8: 15882

10 Chen Z, Turedi B, Abdullah Y A, et al. Single-crystal $\mathrm{MAPbI}_{3}$ perovskite solar cells exceeding $21 \%$ power conversion efficiency. ACS Energy Lett, 2019, 4: 1258-1259

11 Chen J, Morrow D, Fu Y, et al. Single-crystal thin films of cesium lead bromide perovskite epitaxially grown on metal oxide perovskite $\left(\mathrm{SrTiO}_{3}\right)$. J Am Chem Soc, 2017, 139: 13525-13532

12 Zhumekenov A A, Burlakov V M, Saidaminov M I, et al. The role of surface tension in the crystallization of metal halide perovskites. ACS Energy Lett, 2017, 2: 1782-1788

$13 \mathrm{Lv}$ Q, Lian Z, He W, et al. A universal top-down approach toward thickness-controllable perovskite single-crystalline thin films. J Mater Chem C, 2018, 6: 4464-4470

14 Wei P, Wang L, Murali B, et al. Solution-grown monocrystalline hybrid perovskite films for hole-transporter-free solar cells. Adv Mater, 2016, 28: 3383-3390

15 Lei Y, Chen Y, Zhang R, et al. A fabrication process for flexible single-crystal perovskite devices. Nature, 2020, 583: 790-795 